\section{Les Japonais et nous}

Simone Gilgenkrantz
S. Gilgenkrantz : 9, rue Basse, 54330 Clérey-sur-Brénon, France. simsimone.gilgenkrantz@gmail.com

\author{
«Chaque identité est \\ une collectivité d'identités... \\ Chaque identité est, à elle seule, \\ un écosystème... » \\ Jean-Claude Ameisen [7]
}

En buvant du sake (ce qui ne signifie rien d'autre que «boisson alcoolisée»), les Japonais ressentent une sensation déplaisante s'accompagnant d'une rougeur du visage. Nous savons pourquoi depuis 1980 : ils sont près de $50 \%$ à être dépourvus d'une sous-unité d'acétaldéhyde déshydrogénase efficace (ALDH2) - seconde enzyme après l'alcool déshydrogénase dans le métabolisme de l'alcool-remplacée par ALDH2*2, qui est inactive. La présence de l'isoenzyme ALDH2*2 retarde l'oxydation de l'acétaldéhyde et augmente sa concentration dans le sang, d'où ces effets indésirables. Comme nous l'allons voir, cette différence génétique entre Japonais et Européens n'est pas la seule. Celle qui vient d'être récemment découverte dans

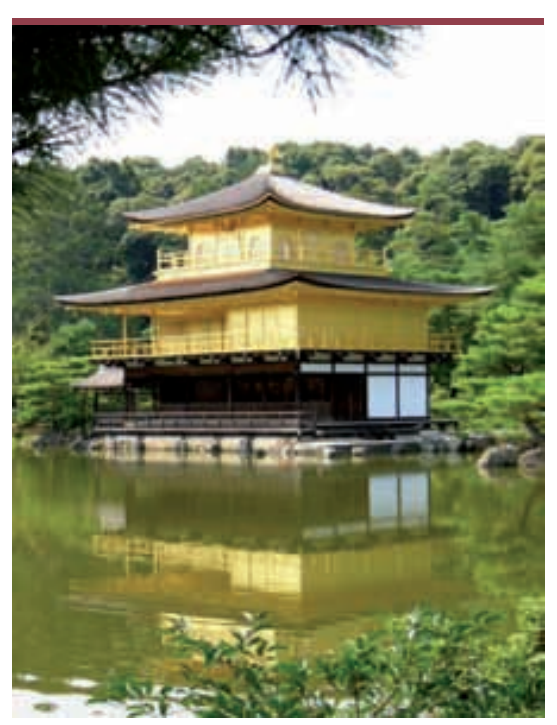

Kinkaku-ji, le pavillon d'or, Kyoto la microflore intestinale, par des étapes successives toutes plus intéressantes les unes que les autres, nous entraîne des rivages de Bretagne, en particulier de I'Unité des végétaux marins et biomolécules de Roscoff $(\rightarrow)$

à ceux du Japon, nous plonge dans des coutumes orientales très

$\rightarrow$ Voir l'article de Mirjam Czjzek et al., page 811 de ce numéro anciennes, nous fait découvrir l'intérêt de la métagénomique, et nous fournit une démonstration exemplaire des étonnants réseaux que les êtres vivants ont tissés entre eux au cours de l'évolution.

\section{Aux délices des sushi}

Le sushi est un des plats emblématiques du Japon. Il semble avoir existé dès le VIII e siècle, le riz étant utilisé pour la conservation du poisson, puis il s'est diversifié. Aujourd'hui, il en existe toutes sortes de variétés. Les makizushi (sushi enroulé) sont parmi les plus classiques et les plus appréciés: le riz au sein duquel se trouvent de fines tranches de légume et de poisson est enroulé dans une feuille de nori (Figure 1).

Nori est le nom japonais pour diverses espèces d'algues comestibles dont se nourrissent les Japonais depuis des siècles : une loi promulguée en 712 mentionne qu'elles doivent faire l'objet d'un impôt. En 987, dans le Utsubo Monogatari (roman d'Utsubo), diverses variétés sont citées. Il s'agit surtout d'algues rouges du genre Porphyra de la famille des Bangiaceae (Figure 2).

\section{Les indispensables algues rouges}

La culture des Porphyra, ces légumes de mer, est pratiquée depuis longtemps. Les asiatiques consomment plus de 10 grammes d'algues par jour ; aux États-

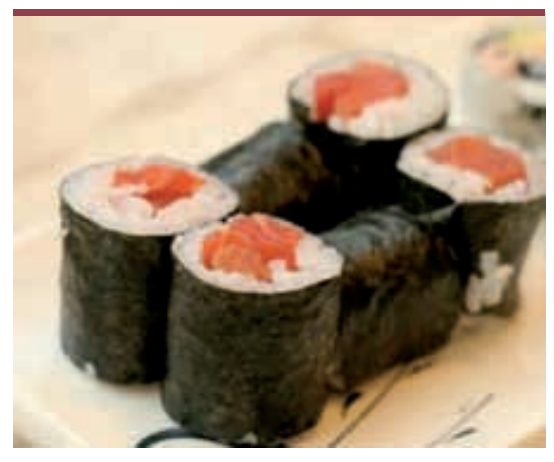

Figure 1. Makizushi.

Unis et en Europe, la multiplication des restaurants japonais et la diffusion de la macrobiotique et de ses modes alimentaires en ont mondialisé la consommation depuis les années 1970. La demande est en constante progression.

C'est pourquoi la culture des Porphyra occupe actuellement 600 kilomètres des eaux côtières japonaises, faisant $d u$ Japon le premier producteur avec plus de 250000 tonnes d'algues fraîches chaque année.

Les diverses variétés de Porphyra sont ensemencées en mer dans des filets (Figure 3).

La récolte débute en décembre pour finir fin mars, début avril, avec plusieurs coupes successives par des moissonneusesbatteuses de la mer. Les algues sont ensuite essorées, étalées sur des cadres et séchées. Puis, selon une ancienne méthode japonaise de la période $\varepsilon_{d o}{ }^{1}$ utilisée pour faire du papier, elles sont broyées et laminées sous forme de feuilles rectangulaires $(18 \times 20 \mathrm{~cm})$ vendues dans le commerce et entrant dans de nombreuses recettes (Figure 4). Ces algues sont très riches en éléments

\footnotetext{
${ }^{1}$ Entre 1600 et 1867.
} 


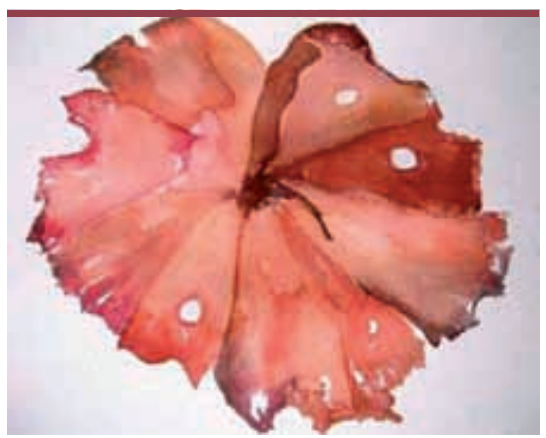

Figure 2. Algue marine du genre Porphyra.

nutritifs (fer, calcium, iode, vitamines et carotène), mais elles contiennent dans leurs parois certains polymères de sucre que la flore intestinale des humains est en principe incapable de digérer.

\section{Stratégie de développement}

\section{de la biomasse marine}

Sur les côtes du Finistère où la récolte du goémon est très ancienne, les Porphyra poussent en abondance, (mais ne peuvent être cultivées en raison de l'importance des marées). À Roscoff, dans la station de biologie marine, des recherches avaient été entreprises pour trouver des protéines capables de transformer la biomasse algale, en particulier les alginates et les carraghénanes, qui constituent une part importante des gélifiants et épaississants $d u$ marché.

Les deux équipes (CNRS/UPMC) impliquées dans cette recherche ont trouvé mieux : une nouvelle catégorie d'enzymes, les $\beta$ porphyranases capables de dégrader la porphyrane, abondante dans les espèces d'algues du genre Porphyra [1]. Car la surface des algues est colonisée par des bactéries marines hétérotrophes qui entretiennent avec les algues des relations trophiques (commensalisme, saprophytisme) et dégradent, par exemple, divers polysaccharides sulfatés grâce à des CAZymes (enzymes carbohydrate actives).

\section{Une nouvelle bactérie marine}

Parmi les bactéries marines, Zobellia galactanivorans (Figure 5) avait été choisie pour ses activités agarolytiques et carraghénolytiques.

Son génome de 5,5 Mb fut séquencé au Genoscope en 2005, puis analysé au MPI (Max Planck Institute) et à I'UMR 7139 de Roscoff. Parmi les glycosides hydrolases (GH) que Zobellia galactanivorans possède, on trouve plusieurs enzymes de la famille GHl6, dont deux d'entre elles n'ont pas d'action sur l'agarose et les carraghénanes, mais sont spécifiquement actives sur la porphyrane. Ces $\beta$ porphyranases constituent donc une nouvelle sous-famille; l'analyse structurale par cristallographie a permis de mettre en évidence le site de reconnaissance du substrat [2].

Grâce à une recherche dans des banques de données, de nombreuses $\beta$ porphyranases ont été trouvées, provenant toutes de bactéries marines, ce qui est parfaitement logique puisque les porphyrases n'existent $(\rightarrow)$ Voir l'article pas dans les plantes de Mirjam Czjzek et terrestres $(\rightarrow) . \quad$ al., page 811 de ce numéro

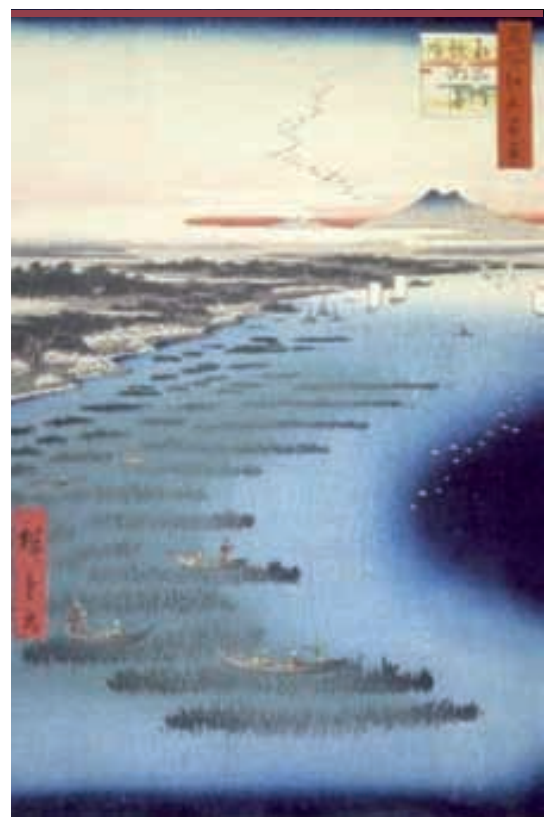

Figure 3. Hiroshige Utagawa (1797-1858). Culture de nori sur le rivage d'0mori, baie de Tokyo (๔ Brooklyn Museum photograph, 2006).

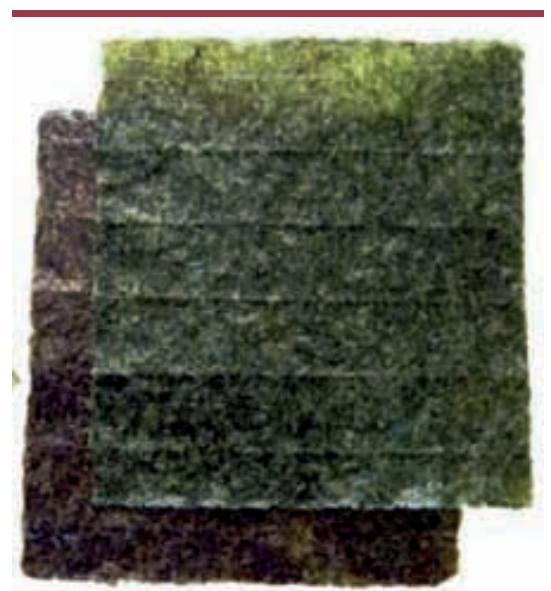

Figure 4. Feuilles de nori prêtes à l'emploi.

Toutefois, la métagénomique réservait une surprise de taille : une bactérie de la flore intestinale humaine était pourvue de porphyranase. II s'agit de Bp 1689 , ou Bacteroïdes plebeius, découverte récemment et formant avec Bacterö̈des coprocola un nouveau groupe de bactéroïdes [3].

\section{La flore intestinale des Japonais}

Cette bactérie a toutefois une particularité : elle n'est présente que dans la flore intestinale des Japonais [4]. Dans une étude comparative entre 13 Japonais et 18 Américains, on observe que seuls les Japonais sont pourvus de porphyranases, mais pas seulement des sujets adultes: un nourrisson japonais - encore nourri au sein - possédait lui aussi des gènes de porphyranases. Bien qu'il soit nécessaire de poursuivre ces recherches sur un plus grand nombre d'individus, il semble que les 0ccidentaux soient dépourvus de Bacteroïdes plebeius.

Comment Bacteroïdes plebeius, un bacille intestinal anaérobie à Gram négatif, $a-t-i l$ pu acquérir cette enzyme? Les chercheurs de Roscoff ont constaté que le génome de cette bactérie nippone contenait le système porphyranolytique complet (6 gènes) que l'on trouve dans les bacteroïdètes marines. II est donc tentant de supposer qu'il résultait d'un transfert horizontal de gènes, phénomène important et fréquent chez les bactéries. 

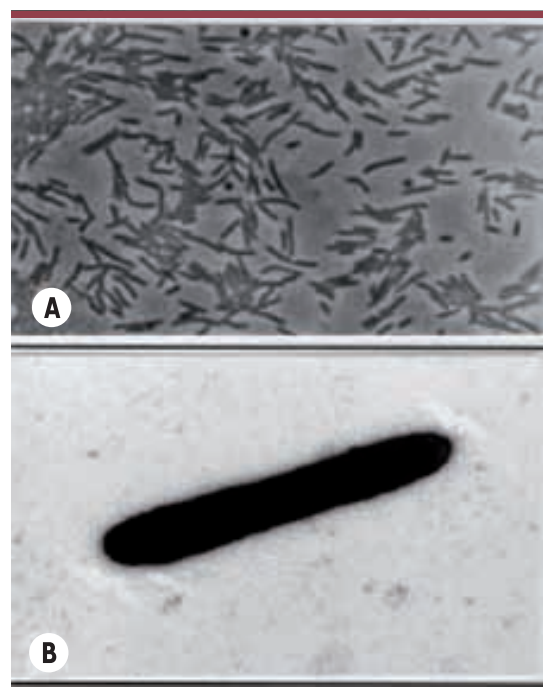

Figure 5. Zobellia galactanivorans. A. Colonies en contraste de phase. B. En microscopie électronique (pas de flagelle visible) [2].

\section{Transfert horizontal de gènes}

Décrit pour la première fois en 1959 par des chercheurs japonais, le transfert horizontal de gènes (THG) démontrait le passage de la résistance aux antibiotiques entre différentes espèces de bactéries [5]. Ce n'est que plus tard que les implications redoutables de cette observation sont apparues en infectiologie. Bien plus surprenant est le transfert de gènes qui s'est produit entre les génomes chloroplastiques et mitochondriaux et le génome nucléaire. Comme indiqué par la théorie endosymbiotique, les mitochondries et les chloroplastes ont probablement comme origine une bactérie endosymbionte d'un ancêtre de la cellule eucaryote. Les THG sont fréquents chez les bactéries et pour les chercheurs de Roscoff, la seule conclusion possible à la présence de cette batterie porphyranolytique est donc bien un transfert horizontal. Les Japonais consomment des algues crues et peuvent donc ingérer des bactéries marines qui se trouvent à leur surface.

À quel moment et comment ce transfert est-il survenu dans cette population insulaire se nourrissant de riz, d'algues (crues) et de poissons depuis des siècles $^{2}$ ? La flore intestinale des coprolithes ou des sujets momifiés pourrait nous éclairer. Outre l'intérêt croissant des variations de flores intestinales selon l'alimentation, en particulier dans les études sur l'obésité [6], et l'exemplarité de l'alimentation nippone qui n'est sans doute pas pour rien dans la longévité des Japonais, ce travail laisse entrevoir l'incroyable réseau que les êtres vivants sont capables de tisser entre eux à travers le temps et l'espace. $\diamond$ The Japanese and us

\footnotetext{
${ }^{2}$ Ce n'est que vers l'époque de la restauration de Meiji, à la fin du XIXe siècle, que la consommation de viande fut permise, voire encouragée.
}

\section{REMERCIEMENTS}

Nous tenons à remercier le professeur Kei Shionoya pour ses informations sur la civilisation japonaise.

\section{CONFLIT D'INTÉRÊTS}

L'auteur déclare n'avoir aucun conflit d'intérêts concernant les données publiées dans cet article.

\section{RÉFÉRENCES}

1. Barbeyron T, L'Haridon S, Corre $\varepsilon$, et al. Zobellia galactanovorans gen. nov., sp. nov., a marine species of Flavobacteriaceae isolated from a red alga, and classification of [Cytophaga] uliginosa (ZoBell and Upham 1944) Reichenbach 1989 as Zobellia uliginosa gen. Int J Syst Evol Microbiol 2001 ; 51 : 985-97.

2. Hehemann JH, Correc G, Barbeyron T, et al. Transfer of carbohydrate-active enzymes from marine bacteria to Japanese gut microbiota. Nature 2010 ; 464 : 908-12.

3. Kitahara M, Sakamoto M, Ike M, et al. Bacteroides plebeius sp. nov. and Bacteroides coprocola sp. nov., isolated from human faeces. Int J Syst Evol Microbiol $2005 ; 55: 2143-7$.

4. Akiba T, Koyama K, Ishiki Y, et al. On the mechanism of the development of multiple-drug-resistant clones of Shigella. Jpn J Microbiol $1960 ; 4: 219-27$.

5. Kurokawa, K, Itoh T, Kuwahara T, et al. Comparative metagenomics revealed commonly enriched gene sets in human gut microbiomes. DNA Res 2007 ; $14: 169-81$.

6. Vijay-Kumar M, Aitken JD, Carvalho FA, et al. Metabolic syndrome and altered gut microbiota in mice lacking Toll-like receptor 5 . Science 2010 ; 328: 228-31.

7. Ameisen JC. Dans la lumière et les ombres. Darwin et le bouleversement du monde. Paris : Fayard/Seuil, 2008: $490 \mathrm{p}$.

8. Czjzek M, Correc G, Michel G. Sommes-nous ce que nous mangeons? Le transfert de gènes du milieu marin vers la microflore intestinale chez les Japonais. Med Sci (Paris) 2010; 26:811-3.

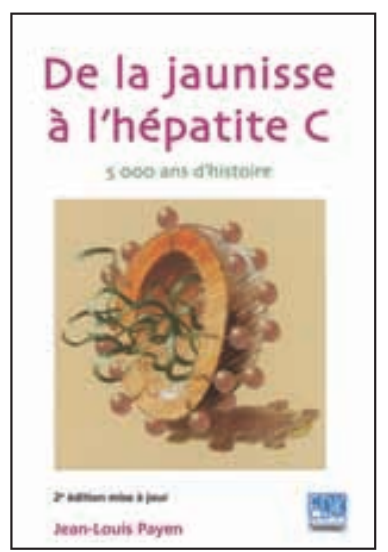

ISBN : 978-2-8425-4136-1 128 pages

\section{Bon de commande}

À retourner à EDK, 2, rue Troyon - 92316 Sèvres Cedex

Tél. : 0155641393 - Fax : 0155641394 - E-mail : edk@edk.fr

NOM : Prénom :

Adresse :

Code postal :

Ville :

Pays :

Fonction :

Je souhaite recevoir l'ouvrage De la jaunisse à l'hépatite $\mathbf{C , ~} \mathbf{5} 000$ ans d'histoire : $12 €+3 €$ de port $=$ $15 €$ TTC

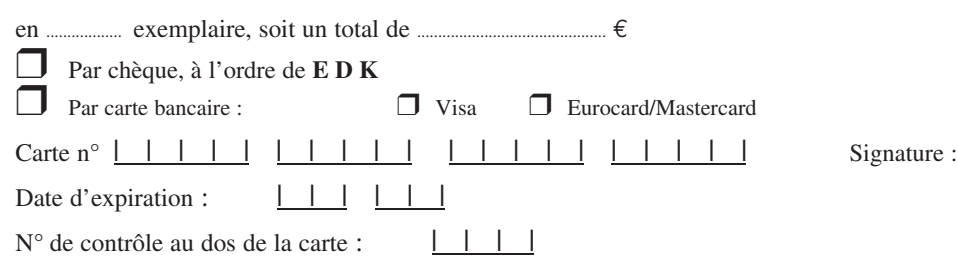

